\title{
$B$ meson light-cone wavefunctions in the heavy quark limit
}

\author{
H. Kawamura ${ }^{a}$, J. Kodairab日, C.-F. Qiao'国, K. Tanakac \\ ${ }^{a}$ Deutsches Elektronen-Synchrotron, DESY \\ Platanenallee 6, D 15738 Zeuthen, Germany \\ ${ }^{\mathrm{b}}$ Department of Physics, Hiroshima University \\ Higashi-Hiroshima 739-8526, Japan \\ ${ }^{c}$ Department of Physics, Juntendo University \\ Inba-gun, Chiba 270-1695, Japan
}

We present a systematic study of the $B$ meson light-cone wavefunctions in QCD in the heavy-quark limit. We construct model-independent formulae for the light-cone wavefunctions in terms of independent dynamical degrees of freedom, which exactly satisfy the QCD equations of motion and constraints from heavy-quark symmetry. The results demonstrate novel behaviors of longitudinal as well as transverse momentum distribution in the $B$ mesons.

Recently systematic methods based on the QCD factorization have been developed for the exclusive $B$ meson decays into light mesons [ 1] (see also Ref. [2]). Essential ingredients in this approach are the light-cone distribution amplitudes for the participating mesons, which express nonperturbative long-distance contribution to the factorized amplitudes. The light-cone distribution amplitudes describe the probability amplitudes to find the meson in a state with the constituents carrying definite light-cone momentum fraction, and thus are process-independent quantity. For the light mesons $\left(\pi, K, \rho, K^{*}\right.$, etc.) appearing in the final state, systematic modelindependent study of the light-cone distributions exists for both leading and higher twists [3]. On the other hand, the light-cone distribution amplitudes for the $B$ mesons are not well-known at present and they provide a major source of uncertainty in the calculations of the decay rates.

By definition, the distribution amplitudes are obtained from light-cone wavefunctions at (almost) zero transverse separation of the con-

\footnotetext{
*Talk presented by K. Tanaka at the International Symposium Radcor 2002 and Loops and Legs 2002, Kloster Banz, September 8-13, 2002.

${ }^{\dagger}$ Supported in part by the Monbu-kagaku-sho Grant-inAid for Scientific Research No.C-13640289.

${ }^{\ddagger}$ Supported by the Grant-in-Aid of JSPS committee.
}

stituents, $\phi(x) \sim \int_{k_{T}^{2}<\mu^{2}} d^{2} k_{T} \Phi\left(x, \boldsymbol{k}_{T}\right)$. The light-cone wavefunctions with transverse momentum dependence are also necessary for computing the power corrections to the exclusive amplitudes, and for estimating the transition form factors for $B \rightarrow D, B \rightarrow \pi$, etc, which constitute another type of long-distance contributions appearing in the factorization approaches for the exclusive $B$ meson decays.

In this work [ 4, 5], we demonstrate that, in the heavy-quark limit relevant for the factorization approaches for the exclusive $B$ meson decays, the $B$ meson light-cone wavefunctions obey exact differential equations, which are based on heavyquark symmetry and the QCD equations of motion. As solution of those differential equations, we derive the model-independent formulae for the light-cone wavefunctions, which involve not only the leading Fock-states with a minimal number of (valence) partons but also the higher Fock-states with additional dynamical gluons.

The light-cone wavefunctions are related to the usual Bethe-Salpeter wavefunctions at equal light-cone time $z^{+}=\left(z^{0}+z^{3}\right) / \sqrt{2}$. In the heavyquark limit, the quark-antiquark light-cone wavefunctions $\tilde{\Phi}_{ \pm}\left(t, z^{2}\right)$ of the $B$ mesons can be introduced in terms of vacuum-to-meson matrix element of nonlocal operators in the the heavy-quark 
effective theory (HQET) [ 6, 5]:

$$
\begin{aligned}
& \left\langle 0\left|\bar{q}(z) \Gamma h_{v}(0)\right| \bar{B}(p)\right\rangle=-\frac{i f_{B} M}{2} \operatorname{Tr}\left[\gamma_{5} \Gamma \frac{1+\not}{2}\right. \\
& \left.\quad \times\left\{\tilde{\Phi}_{+}\left(t, z^{2}\right)-\not z \frac{\tilde{\Phi}_{+}\left(t, z^{2}\right)-\tilde{\Phi}_{-}\left(t, z^{2}\right)}{2 t}\right\}\right]
\end{aligned}
$$

Here $z^{\mu}=\left(0, z^{-}, \boldsymbol{z}_{T}\right), z^{2}=-\boldsymbol{z}_{T}^{2}, v^{2}=1, t=v \cdot z$, and $p^{\mu}=M v^{\mu}$ is the 4-momentum of the $B$ meson with mass $M . h_{v}(x)$ denotes the effective $b$-quark field, $b(x) \approx \exp \left(-i m_{b} v \cdot x\right) h_{v}(x)$, and is subject to the on-shell constraint, $\not h_{v}=h_{v}$ [7]. $\Gamma$ is a generic Dirac matrix and, here and in the following, the path-ordered gauge factors are implied in between the constituent fields. $f_{B}$ is the decay constant defined as usually as $\left\langle 0\left|\bar{q}(0) \gamma^{\mu} \gamma_{5} h_{v}(0)\right| \bar{B}(p)\right\rangle=i f_{B} M v^{\mu}$, so that $\tilde{\Phi}_{ \pm}\left(t=0, z^{2}=0\right)=1$. Eq. (11) is the most general parameterization compatible with Lorentz invariance and the heavy-quark limit.

Higher Fock components in the $B$ mesons are described by multi-particle wavefunctions. We explicitly deal with quark-antiquark-gluon threeparticle wavefunctions, defined as [4]

$$
\begin{aligned}
\left\langle 0\left|\bar{q}(z) g G_{\mu \nu}(u z) z^{\nu} \Gamma h_{v}(0)\right| \bar{B}(p)\right\rangle \\
=\frac{1}{2} f_{B} M \operatorname{Tr}\left[\gamma _ { 5 } \Gamma \frac { 1 + \not p } { 2 } \left\{\left(v_{\mu} \not z-t \gamma_{\mu}\right)\right.\right. \\
\quad \times\left(\tilde{\Psi}_{A}(t, u)-\tilde{\Psi}_{V}(t, u)\right)-i \sigma_{\mu \nu} z^{\nu} \tilde{\Psi}_{V}(t, u) \\
\left.\left.\quad-z_{\mu} \tilde{X}_{A}(t, u)+\frac{z_{\mu}}{t} \not \tilde{Y}_{A}(t, u)\right\}\right]+\ldots,(2)
\end{aligned}
$$

where the ellipses stand for the terms which involve one or more powers of $z^{2}$ and are irrelevant for the present work. We have the four functions $\tilde{\Psi}_{V}, \tilde{\Psi}_{A}, \tilde{X}_{A}$ and $\tilde{Y}_{A}$ as the independent threeparticle wavefunctions in the heavy-quark limit.

The QCD equations of motion impose a set of relations between the above wavefunctions [ 4, 5]. They can be derived most directly from the exact identities between the nonlocal operators:

$$
\begin{aligned}
& \frac{\partial}{\partial x^{\mu}} \bar{q}(x) \gamma^{\mu} \Gamma h_{v}(0) \\
& \quad=i \int_{0}^{1} d u u \bar{q}(x) g G_{\mu \nu}(u x) x^{\nu} \gamma^{\mu} \Gamma h_{v}(0) \\
& v^{\mu} \frac{\partial}{\partial x^{\mu}} \bar{q}(x) \Gamma h_{v}(0)
\end{aligned}
$$

$$
\begin{aligned}
& =i \int_{0}^{1} d u(u-1) \bar{q}(x) g G_{\mu \nu}(u x) v^{\mu} x^{\nu} \Gamma h_{v}(0) \\
& +\left.v^{\mu} \frac{\partial}{\partial y^{\mu}} \bar{q}(x+y) \Gamma h_{v}(y)\right|_{y \rightarrow 0}
\end{aligned}
$$

where $G_{\mu \nu}$ is the gluon field strength tensor, and we have used the equations of motion $\not D q=0$ and $v \cdot D h_{v}=0$ with $D_{\mu}=\partial_{\mu}-i g A_{\mu}$ the covariant derivative. Taking the matrix element with $x_{\mu} \rightarrow$ $z_{\mu}$, the LHS of these identities yield $\tilde{\Phi}_{+}\left(t, z^{2}\right)$, $\tilde{\Phi}_{-}\left(t, z^{2}\right)$ defined in Eq. (11) and their derivatives, $\partial \tilde{\Phi}_{ \pm}\left(t, z^{2}\right) / \partial t$ and $\partial \tilde{\Phi}_{ \pm}\left(t, z^{2}\right) / \partial z^{2}$. The terms in the RHS, which are given by integral of quarkantiquark-gluon operator, are expressed by the three-particle wavefunctions of Eq. (2). The last term of Eq. (4), the derivative over the total translation, yields $\tilde{\Phi}_{ \pm}\left(t, z^{2}\right)$ multiplied by

$\bar{\Lambda}=M-m_{b}=\frac{i v \cdot \partial\left\langle 0\left|\bar{q} \Gamma h_{v}\right| \bar{B}(p)\right\rangle}{\left\langle 0\left|\bar{q} \Gamma h_{v}\right| \bar{B}(p)\right\rangle}$.

This is the usual "effective mass" of meson states in the HQET [7].

Substituting all the Dirac matrices for $\Gamma$, we obtain the four independent constraint equations between the two- and three-particle wavefunctions from Eqs. (3) and (4). We solve this system of equations for the relevant two cases: (i) In the light-cone limit $z^{2} \rightarrow 0\left(\boldsymbol{z}_{T} \rightarrow 0\right)$, with fully taking into account the contribution due to the three-particle wavefunctions. The solution gives exact model-independent representaions for the light-cone distribution amplitudes in terms of independent dynamical degrees of freedom. (ii) For $z^{2} \neq 0$ but in the approximation neglecting the contribution of the three-particle wavefunctions. The solution gives exact analytic formulae for the light-cone wavefunctions with transverse momentum dependence within the valence Fock-states.

Now we discuss the case (i) in detail [4]. In the light-cone limit, the light-cone wavefunctions of Eq. (1) reduce to the light-cone distribution amplitudes $\tilde{\phi}_{ \pm}(t)$ as [ [ $]$

$\tilde{\phi}_{ \pm}(t)=\tilde{\Phi}_{ \pm}\left(t, z^{2}=0\right)$,

and we also introduce the shorthand notations, $\tilde{\phi}_{ \pm}^{\prime}(t) \equiv d \tilde{\phi}_{ \pm}(t) / d t$ and $\partial \tilde{\phi}_{ \pm}(t) / \partial z^{2} \equiv$ $\partial \tilde{\Phi}_{ \pm}\left(t, z^{2}\right) /\left.\partial z^{2}\right|_{z^{2} \rightarrow 0}$, which denote the deriva- 
tives with respect to the longitudinal and transverse separations, respectively. The first identity (3) yields the two equations:

$$
\begin{aligned}
& \tilde{\phi}_{-}^{\prime}(t)-\frac{1}{t}\left(\tilde{\phi}_{+}(t)-\tilde{\phi}_{-}(t)\right) \\
&= 2 t \int_{0}^{1} d u u\left(\tilde{\Psi}_{A}(t, u)-\tilde{\Psi}_{V}(t, u)\right), \quad(7) \\
& \tilde{\phi}_{+}^{\prime}(t)-\tilde{\phi}_{-}^{\prime}(t)-\frac{1}{t}\left(\tilde{\phi}_{+}(t)-\tilde{\phi}_{-}(t)\right)+4 t \frac{\partial \tilde{\phi}_{+}(t)}{\partial z^{2}} \\
&=2 t \int_{0}^{1} d u u\left(\tilde{\Psi}_{A}(t, u)+2 \tilde{\Psi}_{V}(t, u)+\tilde{X}_{A}(t, u)\right),(8)
\end{aligned}
$$

and similarly the second identity (位) yields

$$
\begin{aligned}
\tilde{\phi}_{+}^{\prime}(t) & -\frac{1}{2 t}\left(\tilde{\phi}_{+}(t)-\tilde{\phi}_{-}(t)\right)+i \bar{\Lambda} \tilde{\phi}_{+}(t)+2 t \frac{\partial \tilde{\phi}_{+}(t)}{\partial z^{2}} \\
= & t \int_{0}^{1} d u(u-1)\left(\tilde{\Psi}_{A}(t, u)+\tilde{X}_{A}(t, u)\right),(9) \\
\tilde{\phi}_{+}^{\prime}(t) & -\tilde{\phi}_{-}^{\prime}(t)+\left(i \bar{\Lambda}-\frac{1}{t}\right)\left(\tilde{\phi}_{+}(t)-\tilde{\phi}_{-}(t)\right) \\
+ & 2 t\left(\frac{\partial \tilde{\phi}_{+}(t)}{\partial z^{2}}-\frac{\partial \tilde{\phi}_{-}(t)}{\partial z^{2}}\right) \\
= & 2 t \int_{0}^{1} d u(u-1)\left(\tilde{\Psi}_{A}(t, u)+\tilde{Y}_{A}(t, u)\right) .(10)
\end{aligned}
$$

These Eqs. (7)-(10) are exact in QCD in the heavy-quark limit.

Important observation is that we can eliminate the term $\partial \tilde{\phi}_{+}(t) / \partial z^{2}$ by combining Eqs. (8) and (9). The resulting equation, combined with Eq. (17), gives a system of two differential equations which involve the degrees of freedom along the light-cone only. By going over to the momentum space by $\tilde{\phi}_{ \pm}(t)=\int d \omega e^{-i \omega t} \phi_{ \pm}(\omega)$, and $\tilde{F}(t, u)=\int d \omega d \xi e^{-i(\omega+\xi u) t} F(\omega, \xi)$ with $F=$ $\left\{\Psi_{V}, \Psi_{A}, X_{A}, Y_{A}\right\}$, the corresponding differential equations read [ $[$ ]

$$
\begin{aligned}
\omega \frac{d \phi_{-}(\omega)}{d \omega} & +\phi_{+}(\omega)=I(\omega), \\
(\omega-2 \bar{\Lambda}) \phi_{+}(\omega) & +\omega \phi_{-}(\omega)=J(\omega),
\end{aligned}
$$

where $I(\omega)$ and $J(\omega)$ denote the "source" terms due to three-particle wavefunctions as

$I(\omega)=2 \frac{d}{d \omega} \int_{0}^{\omega} d \rho \int_{\omega-\rho}^{\infty} \frac{d \xi}{\xi} \frac{\partial}{\partial \xi}\left[\Psi_{A}(\rho, \xi)-\Psi_{V}(\rho, \xi)\right]$,

$$
\begin{aligned}
J(\omega) & =-2 \frac{d}{d \omega} \int_{0}^{\omega} d \rho \int_{\omega-\rho}^{\infty} \frac{d \xi}{\xi}\left[\Psi_{A}(\rho, \xi)+X_{A}(\rho, \xi)\right] \\
& -4 \int_{0}^{\omega} d \rho \int_{\omega-\rho}^{\infty} \frac{d \xi}{\xi} \frac{\partial \Psi_{V}(\rho, \xi)}{\partial \xi} .
\end{aligned}
$$

Eqs. (11), (12) can be solved for $\phi_{+}(\omega)$ and $\phi_{-}(\omega)$. The boundary conditions are specified as $\phi_{ \pm}(\omega)=0$ for $\omega<0$ or $\omega \rightarrow \infty$, because $\omega v^{+}$has the meaning of the light-cone projection $k^{+}$of the light-antiquark momentum in the $B$ meson, and the normalization condition is $\int_{0}^{\infty} d \omega \phi_{ \pm}(\omega)=\tilde{\Phi}_{ \pm}(0,0)=1$. Obviously, the solution can be decomposed into two pieces as

$\phi_{ \pm}(\omega)=\phi_{ \pm}^{(W)}(\omega)+\phi_{ \pm}^{(g)}(\omega)$,

where $\phi_{ \pm}^{(W)}(\omega)$ are the solution with $I(\omega)=$ $J(\omega)=0$, which corresponds to the "WandzuraWilczek approximation [ [3]" $\Psi_{V}=\Psi_{A}=X_{A}=$ $Y_{A}=0 . \phi_{ \pm}^{(g)}(\omega)$ denote the pieces induced by the source terms $I(\omega)$ and $J(\omega)$.

We are able to obtain the analytic solution for the Wandzura-Wilczek part as

$\phi_{ \pm}^{(W)}(\omega)=\frac{\bar{\Lambda} \pm(\omega-\bar{\Lambda})}{2 \bar{\Lambda}^{2}} \theta(\omega) \theta(2 \bar{\Lambda}-\omega)$.

Moreover, the solution for $\phi_{ \pm}^{(g)}$ can be obtained straightforwardly, and reads $(\omega \geq 0)$ :

$$
\begin{aligned}
\phi_{+}^{(g)}(\omega)= & \frac{\omega}{2 \bar{\Lambda}} \mathcal{G}(\omega), \\
\phi_{-}^{(g)}(\omega)= & \frac{2 \bar{\Lambda}-\omega}{2 \bar{\Lambda}} \mathcal{G}(\omega)+\frac{J(\omega)}{\omega}, \\
\mathcal{G}(\omega)= & \theta(2 \bar{\Lambda}-\omega)\left\{\int_{0}^{\omega} d \rho \frac{K(\rho)}{2 \bar{\Lambda}-\rho}-\frac{J(0)}{2 \bar{\Lambda}}\right\} \\
& -\theta(\omega-2 \bar{\Lambda}) \int_{\omega}^{\infty} d \rho \frac{K(\rho)}{2 \bar{\Lambda}-\rho} \\
& -\int_{\omega}^{\infty} d \rho\left(\frac{K(\rho)}{\rho}+\frac{J(\rho)}{\rho^{2}}\right),
\end{aligned}
$$

with $K(\rho)=I(\rho)+[1 /(2 \bar{\Lambda})-d / d \rho] J(\rho)$. The solution (14) with Eqs. (15)-(18) is exact, and reveals that $\phi_{ \pm}$contain the three-particle contributions. This is also visualized explicitly in terms of the Mellin moments $\left\langle\omega^{n}\right\rangle_{ \pm} \equiv \int d \omega \omega^{n} \phi_{ \pm}(\omega)$ $(n=0,1,2, \cdots)$. Here we present some examples for a few low moments: $\langle\omega\rangle_{ \pm}=(3 \pm 1) \bar{\Lambda} / 3$, and

$$
\left\langle\omega^{2}\right\rangle_{ \pm}=\frac{4 \pm 2}{3} \bar{\Lambda}^{2}+\frac{1}{3} \lambda_{E}^{2}+\frac{1}{3} \lambda_{H}^{2} \pm \frac{1}{3} \lambda_{E}^{2},
$$


where $\lambda_{E}$ and $\lambda_{H}$ are due to $\phi_{ \pm}^{(g)}$, and are related to the chromoelectric and chromomagnetic fields in the $B$ meson rest frame as $\left\langle 0\left|\bar{q} g \boldsymbol{E} \cdot \boldsymbol{\alpha} \gamma_{5} h_{v}\right| \bar{B}(\boldsymbol{p}=0)\right\rangle=f_{B} M \lambda_{E}^{2},\langle 0| \bar{q} g \boldsymbol{H}$. $\boldsymbol{\sigma} \gamma_{5} h_{v}|\bar{B}(\boldsymbol{p}=0)\rangle=i f_{B} M \lambda_{H}^{2}$. Our solution (14) allows us to further derive the analytic formulae for the general moment $n$ in terms of matrix element of local two- and three-particle operators with dimension $n+3$ (see Ref. [四] for the detail).

The behavior of Eq. (11) for a fast-moving meson, $t=v \cdot z \rightarrow \infty$, shows that $\phi_{+}$is of leading-twist whereas $\phi_{-}$has subleading twist; the three-particle contributions to the leadingtwist $\phi_{+}$are in contrast with the case of the light mesons [ 3], where the leading-twist amplitudes correspond to the valence Fock component, while the higher-twist amplitudes involve multiparticle states. We note that there exists an estimate $\lambda_{E}^{2} / \bar{\Lambda}^{2}=0.36 \pm 0.20, \lambda_{H}^{2} / \bar{\Lambda}^{2}=0.60 \pm 0.23$ by QCD sum rules [ 8] (see Eq. (19)). This might suggest that, in the $B$ mesons, the threeparticle contributions could play important roles even in the leading twist level. In this connection, the shape of our Wandzura-Wilczek contributions (15), which are determined uniquely in analytic form in terms of $\bar{\Lambda}$, is rather different from various "model" distribution amplitudes that have been used in the existing literature: One exapmle of such models is $\phi_{+}^{G N}(\omega)=$ $\left(\omega / \omega_{0}^{2}\right) e^{-\omega / \omega_{0}}$ and $\phi_{-}^{G N}(\omega)=\left(1 / \omega_{0}\right) e^{-\omega / \omega_{0}}$ with $\omega_{0}=2 \bar{\Lambda} / 3$, inspired by the QCD sum rule estimates [ 8]. These have very different shape compared with Eq. (15), except the behavior $\phi_{+}^{G N}(\omega) \sim \omega, \phi_{-}^{G N}(\omega) \sim$ const, as $\omega \rightarrow 0$.

Next we proceed to the case (ii), where we get

$$
\begin{aligned}
& \omega \frac{\partial \Phi_{-}}{\partial \omega}+\Phi_{+}+z^{2} \frac{\partial}{\partial z^{2}}\left(\Phi_{+}-\Phi_{-}\right)=0 \\
& \left(\omega \frac{\partial}{\partial \omega}+2\right)\left(\Phi_{+}-\Phi_{-}\right)+4 \frac{\partial^{2}}{\partial \omega^{2}} \frac{\partial \Phi_{+}}{\partial z^{2}}=0 \\
& {\left[(\omega-\bar{\Lambda}) \frac{\partial}{\partial \omega}+\frac{3}{2}\right] \Phi_{+}-\frac{1}{2} \Phi_{-}+2 \frac{\partial^{2}}{\partial \omega^{2}} \frac{\partial \Phi_{+}}{\partial z^{2}}=0} \\
& {\left[(\omega-\bar{\Lambda}) \frac{\partial}{\partial \omega}+2\right]\left(\Phi_{+}-\Phi_{-}\right)} \\
& +2 \frac{\partial^{2}}{\partial \omega^{2}}\left(\frac{\partial \Phi_{+}}{\partial z^{2}}-\frac{\partial \Phi_{-}}{\partial z^{2}}\right)=0
\end{aligned}
$$

corresponding to Eqs. (7), (8), (9), (10), re- spectively. Here Eqs. (20)-(23) are given in the " $\omega$-representation" $\Phi_{ \pm} \equiv \Phi_{ \pm}\left(\omega, z^{2}\right)$, instead of the "t-representation", via $\tilde{\Phi}_{ \pm}\left(t, z^{2}\right)=$ $\int d \omega e^{-i \omega t} \Phi_{ \pm}\left(\omega, z^{2}\right)$. The light-cone limit is not taken so that the terms proportional to $z^{2}$ appear in Eq. (20). Note that we have neglected the contribution from the quark-antiquark-gluon three-particle operators.

By combining Eqs. (21) and (22), we eliminate the last term in their LHS. The resulting equation can be integrated with boundary conditions $\Phi_{ \pm}\left(\omega, z^{2}\right)=0$ for $\omega<0$ or $\omega \rightarrow \infty$ as

$(\omega-2 \bar{\Lambda}) \Phi_{+}+\omega \Phi_{-}=0$.

In the limit $z^{2} \rightarrow 0$, Eqs. (20) and (24) reduce to Eqs. (11) and (12) in the Wandzura-Wilczek approximation. The corresponding solution (15) serves as "boundary conditions" to solve Eqs. (20)-(23) for $z^{2} \neq 0$. Then, from Eqs. (20) and (24), we find, as the solution in the WandzuraWilczek approximation for $z^{2} \neq 0$,

$\Phi_{ \pm}^{(W)}\left(\omega, z^{2}\right)=\phi_{ \pm}^{(W)}(\omega) \xi\left(z^{2} \omega(2 \bar{\Lambda}-\omega)\right)$.

Here $\xi(x)$ is a function of a single variable $x$, and can be determined from a remaining differential equation (22) or (23) as $\xi(x)=$ $J_{0}(\sqrt{-x})$ where $J_{0}$ is a (regular) Bessel function. This result gives analytic solution for the lightcone wavefunctions with the transverse separation $\boldsymbol{z}_{T}^{2}=-z^{2}$. The momentum-space wavefunctions $\Phi_{ \pm}^{(W)}\left(\omega, \boldsymbol{k}_{T}\right)$, defined by $\tilde{\Phi}_{ \pm}^{(W)}\left(t,-\boldsymbol{z}_{T}^{2}\right)=$ $\int d \omega d^{2} k_{T} e^{-i \omega t+i \boldsymbol{k}_{T} \cdot \boldsymbol{z}_{T}} \Phi_{ \pm}^{(W)}\left(\omega, \boldsymbol{k}_{T}\right)$, read

$\Phi_{ \pm}^{(W)}\left(\omega, \boldsymbol{k}_{T}\right)=\frac{\phi_{ \pm}^{(W)}(\omega)}{\pi} \delta\left(\boldsymbol{k}_{T}^{2}-\omega(2 \bar{\Lambda}-\omega)\right)$.

The result (26) gives exact description of the valence Fock components of the $B$ meson wavefunctions in the heavy-quark limit, and represent their transverse momentum dependence explicitly. These results show that the dynamics within the two-particle Fock states is determined solely in terms of a single nonperturbative parameter $\bar{\Lambda}$.

The transverse momentum distributions in the $B$ mesons have been unknown, so that various models have been used in the literature. Frequently used models assume complete separation (factorization) between the longitudinal and 
transverse momentum-dependence in the wavefunctions (see e.g. Refs. [2, 6]). A typical example of such models [2] is given by $\Phi_{ \pm}^{K L S}\left(\omega, \boldsymbol{k}_{T}\right)=$ $N \omega^{2}(1-\omega)^{2} e^{-\omega^{2} /\left(2 \beta^{2}\right)} \times e^{-\boldsymbol{k}_{T}^{2} /\left(2 \kappa^{2}\right)}$ with some constants $N, \beta$, and $\kappa$. Eq. (26) shows that transverse and longitudinal momenta are strongly correlated through the combination $\boldsymbol{k}_{T}^{2} /[\omega(2 \bar{\Lambda}-\omega)]$, therefore the "factorization models" are not justified. We further note that many models assume Gaussian distribution for the $\boldsymbol{k}_{T}$-dependence as in $\Phi_{ \pm}^{K L S}$. These models show strong dumping at large $\left|\boldsymbol{z}_{T}\right|$ as $\sim \exp \left(-\kappa^{2} \boldsymbol{z}_{T}^{2} / 2\right)$. In contrast, our wavefunctions 25) have slow-damping with oscillatory behavior as $\Phi_{ \pm}^{(W)}\left(\omega,-z_{T}^{2}\right) \sim$ $\cos \left(\left|\boldsymbol{z}_{T}\right| \sqrt{\omega(2 \bar{\Lambda}-\omega)}-\pi / 4\right) / \sqrt{\left|\boldsymbol{z}_{T}\right|}$.

Finally, we can estimate the effects neglected in our solution (26). Inspecting the $t \rightarrow 0$ limit of Eqs. (8), (10), one immediately obtains the exact result for $\partial \tilde{\phi}_{ \pm}(0) / \partial z^{2}$, which gives the first moment of $\boldsymbol{k}_{T}^{2}$ as [ 5

$\int d \omega d^{2} k_{T} \boldsymbol{k}_{T}^{2} \Phi_{ \pm}\left(\omega, \boldsymbol{k}_{T}\right)=\frac{2}{3}\left(\bar{\Lambda}^{2}+\lambda_{E}^{2}+\lambda_{H}^{2}\right)$

with $\lambda_{E}$ and $\lambda_{H}$ of Eq. (19). Here $\Phi_{ \pm}=\Phi_{ \pm}^{(W)}+$ $\Phi_{ \pm}^{(g)}$ denote the total wavefunctions which include the higher Fock contributions $\Phi_{ \pm}^{(g)}$ induced by the three-particle operators. From Eq. (26), we get $\int d \omega d^{2} k_{T} \boldsymbol{k}_{T}^{2} \Phi_{ \pm}^{(W)}\left(\omega, \boldsymbol{k}_{T}\right)=2 \bar{\Lambda}^{2} / 3$, so that the terms $2\left(\lambda_{E}^{2}+\lambda_{H}^{2}\right) / 3$ of Eq. 277) come from $\Phi_{ \pm}^{(g)}$. The result (27), combined with a QCD sum rule estimate of $\lambda_{E}, \lambda_{H}$ mentioned below Eq. (19), suggests that the higher Fock contributions might considerably broaden the transverse momentum distribution. However, qualitative features discussed above, like non-factorization of longitudinal and transverse directions, "slow-damping" for transverse directions, etc., will be unaltered by the effects of multi-particle states.

To summarize, we have derived a system of differential equations for the $B$ meson light-cone wavefunctions and obtained the corresponding analytic solution. The differential equations are derived from the exact equations of motion of QCD in the heavy-quark limit. The heavy-quark symmetry plays an essential role by reducing the number of independent wavefunctions drastically, so that the configuration of quark and antiquark in the $B$ mesons is described by only two lightcone wavefunctions. As a result, a system of four differential equations from the equations of motion allows us to obtain model-independent formulae of these two wavefunctions, which reveal roles of the leading Fock-states, as well as the higher Fock-states with additional dynamical gluons. Also due to the power of heavyquark symmetry, our Wandzura-Wilczek parts (15), (26), which correspond to the leading Fockstates, are given in simple analytic formulae involving one single nonperturbative parameter $\bar{\Lambda}$. Heavy-quark symmetry also guarantees that our solutions determine the light-cone wavefunctions for the $B^{*}$ mesons and also for the $D, D^{*}$ mesons in the heavy-quark limit.

We emphasize that our solutions provide the powerful framework for building up the $B$ meson light-cone wavefunctions and their phenomenological applications, because the solutions satisfy all relevant QCD constraints. Further developments like those required to clarify the effects of multi-particle states can be exploited systematically starting from the exact results in this work.

\section{REFERENCES}

[1] M. Beneke et al., Nucl. Phys. B591 (2000) 313; B606 (2001) 245; C. W. Bauer, D. Pirjol and I. W. Stewart, Phys. Rev. Lett. 87 (2001) 201806; G. Buchalla, in these proceedings.

[2] Y. Y. Keum, H. Li and A. I. Sanda, Phys. Rev. D63 (2001) 054008.

[3] V. M. Braun and I. E. Filyanov, Z. Phys. C48, 239 (1990); P. Ball et al., Nucl. Phys. B529 (1998) 323.

[4] H. Kawamura, J. Kodaira, C.F. Qiao and K. Tanaka, Phys. Lett. B523 (2001) 111; Erratum-ibid. B536 (2002) 344.

[5] H. Kawamura, J. Kodaira, C.F. Qiao and K. Tanaka, hep-ph/0112174.

[6] S. Descotes-Genon and C. T. Sachrajda, Nucl. Phys. B625 (2002) 239.

[7] M. Neubert, Phys. Rept. 245 (1994) 259.

[8] A. G. Grozin and M. Neubert, Phys. Rev. D55 (1997) 272. 